\title{
Ubiquitin-Conjugating Enzyme E2 A
}

National Cancer Institute

\section{Source}

National Cancer Institute. Ubiquitin-Conjugating Enzyme E2 A. NCI Thesaurus. Code C106302.

Ubiquitin-conjug ating enzyme E2 A (152 aa, $\sim 17 \mathrm{kDa}$ ) is encoded by the human UBE2A gene. This protein is involved in the monoubiquitination of histone $\mathrm{H} 2 \mathrm{~B}$. 